\title{
Optimal Design and Control Simulation of a Monolithic Piezoelectric Microactuator with Integrated Sensor
}

\author{
Roba El Khoury Moussa, Mathieu Grossard, Nicolas Chaillet, Member IEEE, Mehdi Boukallel, \\ and Arnaud Hubert
}

\begin{abstract}
This paper presents an important evolution in an optimization method, called FlexIn, developed for the optimal design of piezoelectric compliant micromechanisms. It is based on a flexible building block method that uses an evolutionary approach, to optimize a truss-like planar structure made of piezoelectric passive, active and now sensitive building blocks. The model of these blocks is established by means of a finiteelement electromechanical formulation. The main contribution of this paper is to present an new control-observation-oriented criterion considered in the optimization procedure, among other conventional mechanical criteria, to optimize modal observability of the structure, for the placement of piezoactuating and piezosensing parts. In order to point out the underlying interests of this method for the design of smart structures with integrated actuators and sensors, a planar piezoelectric compliant smart micro-mechanism is optimally synthesized. Simulations of the device are finally performed illustrating the role of the observation-oriented criterion in simplifying the control of such smart structures.
\end{abstract}

\section{INTRODUCTION}

I $\mathrm{N}$ microrobotic applications, compliant active materials are widely used for miniaturization and assembly purposes. One interesting type of smart material used in such matter is the piezoceramic PZT. Beyond their appealing properties in the sense of micromechatronic design, an underlying advantage of piezoelectric materials is the reversibility of their electromechanical coupling effect, which explains their potential use in microrobotic applications as actuators and/or sensors [1], [2], [3], etc. Furthermore, due to their high energy density, compactness, and high bandwidth, piezoceramic actuators and sensors are used for many highfrequency applications.

Moreover, some researches deal with self-sensing actuation, where the piezoelectric material is used simultaneously for actuation and sensing purposes, on simple piezoelectric structures with no placement optimization involved [4], [5]. Numerous papers only address the problem of optimally placing piezoelectric actuator and sensor parts within flexible

R. El Khoury Moussa is with both Femto-st Institute, Université de Franche Comté, Besançon, F-25000 France, and also with CEA, LIST, Interactive Robotics Laboratory, Fontenay-aux-Roses, F-92265, France; roba.el-khoury-moussa@cea.fr

M. Grossard is with CEA, LIST, Interactive Robotics Laboratory, Fontenay-aux-Roses, F-92265, France; mathieu.grossard@cea. fr

$\mathrm{N}$. Chaillet and A. Hubert are with the Femto-st Institute Besançon, F-25000 France; nicolas. chaillet@femto-st. fr and arnaud. hubertefemto-st. fr

M. Boukallel is with CEA, LIST, Sensory and Ambiant Interfaces Laboratory, Fontenay-aux-Roses, F-92265, France; mehdi.boukalleldcea.fr structures, in order to achieve active vibration control and to minimize the spillover effects [6], [7], [8]. These studies are based on controllability and observability modal analysis of the compliant structure, performed separately for the optimization of actuator and sensor placement respectively. One drawback can be that a "well-observed" mode can have a low control authority and vice-versa.

In order to overcome this problem and thus ensure efficient structure control, we propose a new optimization criterion that takes into account both controllability and observability characteristics. This metric is integrated in a more global systematic design approach, based on topology optimization of the structure, as well as that of its frequency responses, to design compliant micro-mechanisms. In this method, called FlexIn (Flexible Innovation) [9], based on the flexible building block method, we propose to optimally integrate the piezoelectric sensor part in the mechanical piezoelectric microactuator. To do so, we use two criteria that can help the designer to optimally place the piezoelectric part. The first one considers purely static electromechanical aspect [10], while the second one is based on modal controllability and observability analysis as briefly cited above. More generally, this last criterion is a useful tool that can address several problems. In addition of avoiding noise amplification [11], and spillover-effects [12], the main purpose is to guarantee that the flexible modes involved in the response of the deflexion are well-observed by the sensing part of the structure.

In this paper, we will first briefly review the underlying idea of the FlexIn method, based on a finite element (FE) formulation, for the design of smart compliant mechanisms (section II). In section III, the state-space approach of the structure is presented in the modal base. Taking advantage of this particular representation form, a gramian interpretation is drawn to take into account, in the optimization algorithm, controllability aspect for actuators and observability aspect for sensors in flexible structures, by means of a new numerical criterion. The latter ensures dynamic performances required to optimally integrate the piezoactive and piezosensing parts within the compliant smart mechanism. The last part of the paper introduces the optimization and simulations results of one chosen pseudo-optimal solution. It demonstrates the interest of such a method and observabilityoriented criterion in the design of microrobotic structures. 


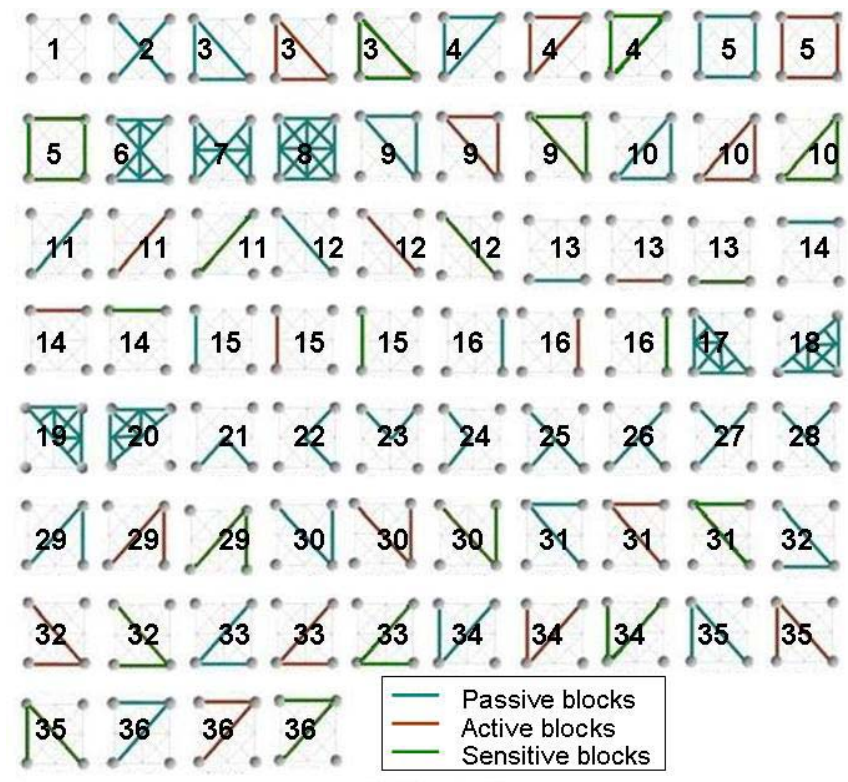

Fig. 1. Passive (blue), piezoactuating (red) and piezosensitive (green) libraries of compliant building blocks, for planar compliant mechanisms synthesis using FlexIn.

\section{FLEXIN: A COMPLIANT MECHANISMS STOCHASTIC DESIGN METHODOLOGY}

\section{A. Principles of the Method}

FlexIn is an optimization software for the design of planar compliant micromechanisms. It is based on a multiobjective genetic algorithm approach, which consists of searching for an optimal distribution of allowed building blocks. The resulting structure is thus an assembly of elementary passive, active or sensitive compliant blocks chosen in three different libraries (Fig. 1), for simply mechanical, actuation and sensing functions respectively. In addition to topological specifications, the optimization problem appoints an optimal set of boundary conditions (fixed frame location, contacts, actuators, sensors, end-effectors, etc.), dimensions and materials, based on the optimization criteria selected by the designer. More detailed descriptions of the method can be found in [9] and [13].

\section{B. Electromechanical FE Model of the Piezoelectric Struc- tures}

In the optimization procedure, the computation of different criteria requires the FE model of each block of the libraries. To obtain the FE formulation of the piezoelectric blocks, a model of a piezoelectric beam is needed first, exploiting both direct and inverse piezoelectricity effects separately for sensing and actuation purposes respectively (Fig. 2). More detailed explanations for the piezoelectric actuation model are given in [13]. The piezoelectric sensing model can be well understood from [10].

The piezoelectric blocks' characteristic matrices (mass, stiffness, electromechanical coupling and electric capacity) are calculated at the beginning of the optimization algorithm,

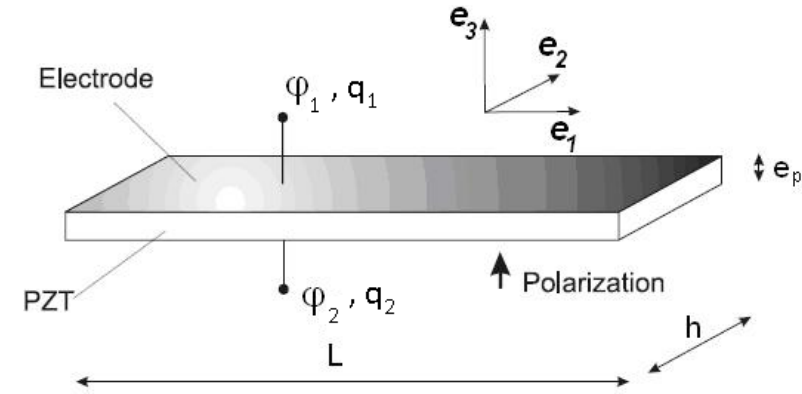

Fig. 2. Thickness-polarized piezoelectric beam transducer with electroded surfaces, and orientation in the material reference frame $\left(\mathbf{e}_{1}, \mathbf{e}_{2}, \mathbf{e}_{3}\right) .\left(\varphi_{1}\right.$, $\left.\varphi_{2}\right)$ and $\left(q_{1}, q_{2}\right)$ denote respectively the electric potential for actuation case, and the electric charges for sensing case, of the two electrodes.

by the association of the corresponding beam matrices in the global coordinate system of the structure.

Each flexible structure synthesized using blocks by FlexIn, is then defined as a finite-dimensional linear system modelled by:

$$
\begin{aligned}
& \mathbf{M}_{\mathbf{g}} \ddot{\eta}_{\mathrm{g}}+\mathbf{K}_{\mathbf{g}} \eta_{\mathrm{g}}=\mathbf{E}_{\mathbf{g}} \mathbf{u} \\
& \mathbf{y}_{\mathbf{c o}}=\delta=\mathbf{F}_{\mathbf{g}} \eta_{\mathrm{g}} \\
& \mathbf{y}_{\mathbf{o b}}=\mathbf{q}_{\mathbf{g}}=\mathbf{L}_{\mathbf{g}} \eta_{\mathrm{g}}
\end{aligned}
$$

The foregoing second-order differential matrix equations represent the undamped dynamic behaviour of such a system, where $\mathbf{M}_{\mathrm{g}}$ and $\mathbf{K}_{\mathrm{g}}$ are the structure's mass and stiffness matrices respectively, obtained by the assembly of the matrices of all the blocks constituting the structure. Considering the integers $p, s$, and $r$, as respectively the numbers of DOFs of the structure, number of inputs (i.e. actuators), and number of tip displacement outputs, $\eta_{\mathbf{g}}$ is then the $p \times 1$ nodal displacement vector and $\mathbf{u}$ is the $s \times 1$ input vector. The $p \times s$ input matrix $\mathbf{E}_{\mathbf{g}}$ reflects the location of the actuated DOFs, while $\mathbf{y}_{\text {co }}$ is the $r \times 1$ controlled output vector representing the output tip displacement $\delta$ through $r \times p$ output displacement matrix $\mathbf{F}_{\mathbf{g}}$. The third equation expresses the electric charges $\left(\mathbf{y}_{\mathbf{o b}}=\mathbf{q}_{\mathrm{g}}\right)$ obtained by the integrated sensing function from the direct piezoelectric effect. Note that $\mathbf{L}_{\mathbf{g}}$ is the $1 \times p$ single output matrix indicating the placement of piezoelectric sensor in the structure. Hence, it is important to note that controlled output variable $\left(\mathbf{y}_{\mathbf{c o}}\right)$ is not the observed output one ( $\left.\mathbf{y}_{\mathbf{o b}}\right)$, as in usual other microrobotic systems, where the controlled tip of the piezocantilever is observed through external sensor.

\section{A NEW CRITERION FOR MATCHING OBSERVABILITY AND HIGH CONTROL AUTHORITY OPTIMIZATION}

In order to successfully achieve suitable dynamic openloop performances (see section III.C), an optimal topology design strategy is derived taking into account controlobservability criterion. The latter based on modal balanced gramians and observability gramians interpretations will be defined to help optimally integrating actuators and sensors in the microstructure. To do so, the physical coordinate base representation (1) is firstly transformed in the modal base 


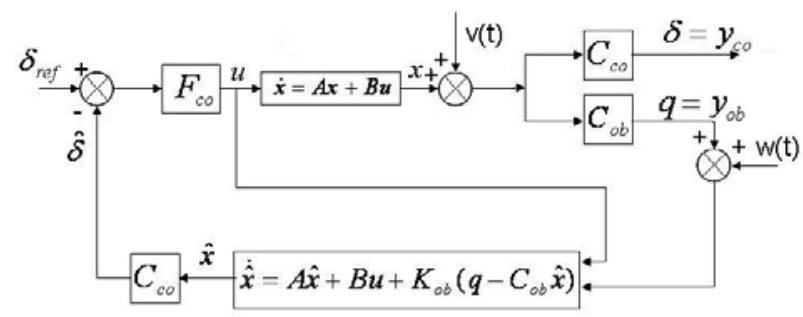

Fig. 3. Control-observation diagram for compliant mechanism with integrated piezoactuator and piezosensor where $F_{c o}$ and $K_{o b}$ are the control and observator gains respectively.

to display the flexible modes. Then, we design an observer that will estimate the flexible modes included in the state vector through the measured electric charge vector $\mathbf{q}_{\mathrm{g}}$ on the sensing blocks of our optimal device. Finally we propose to control the output tip displacement $\delta$ through a state feedback corrector.

\section{A. Modal Representation for Flexible Structures}

By means of modal decomposition, and solving the free undamped vibration eigenproblem, the first equation in (1) leads to the eigenvectors matrix $\boldsymbol{\Psi}$ and natural frequencies $\omega_{i}$ of the system. We then obtain:

$$
\begin{aligned}
& \ddot{\mathbf{z}}+\operatorname{diag}\left(\omega_{i}^{2}\right) \mathbf{z}=\mathbf{\Psi}^{t} \mathbf{E}_{\mathbf{g}} \mathbf{u} \\
& \mathbf{y}_{\mathbf{c o}}=\delta=\mathbf{F}_{\mathbf{g}} \Psi_{\mathbf{z}} \\
& \mathbf{y}_{\mathbf{o b}}=\mathbf{q}_{\mathbf{g}}=\mathbf{L}_{\mathbf{g}} \Psi_{\mathbf{z}}
\end{aligned}
$$

where $\mathbf{z}$ is the $p \times 1$ modal displacement vector.

By introducing modal damping using Basil's hypothesis, the first equation in (2) becomes:

$$
\ddot{\mathbf{z}}+\operatorname{diag}\left(2 \xi_{\mathrm{i}} \omega_{i}\right) \dot{\mathbf{z}}+\operatorname{diag}\left(\omega_{i}^{2}\right) \mathbf{z}=\Psi^{t} \mathbf{E}_{\mathbf{g}} \mathbf{u}
$$

where $\xi_{i}$ is the $i^{\text {th }}$ modal damping ratio.

One interesting $2 p \times 1$ state vector $\mathbf{x}$ typically used for flexible structures, and whose advantages are revealed in [14], consists of modal velocities and frequency-weighted modal displacements:

$$
\mathbf{x}=\left(\begin{array}{ccccc}
\dot{z}_{1} & \omega_{1} z_{1} & \ldots & \dot{z}_{p} & \omega_{p} z_{p}
\end{array}\right)^{t}
$$

Since controlled and observed output vectors are not the same, the modal state-space representation can be written as follows:

$$
\begin{aligned}
& \dot{\mathrm{x}}=\mathbf{A x}+\mathbf{B u}, \\
& \mathbf{y}_{\mathbf{c o}}=\mathbf{C}_{\mathbf{c o}} \mathbf{x}, \\
& \mathbf{y}_{\mathrm{ob}}=\mathbf{C}_{\mathbf{o b}} \mathbf{x} .
\end{aligned}
$$

which leads to two matrices triplets $\left(\mathbf{A}, \mathbf{B}, \mathbf{C}_{\mathbf{c o}}\right)$ and $\left(\mathbf{A}, \mathbf{B}, \mathbf{C}_{\mathbf{o b}}\right)$ designating the control and observation state space models respectively.

The matrices take the forms $\mathbf{A}=\operatorname{diag}\left(\mathbf{A}_{\mathbf{1}}, \ldots \mathbf{A}_{\mathbf{p}}\right)$, $\mathbf{B}=\left(\mathbf{B}_{1}^{t}, \ldots \mathbf{B}_{\mathbf{p}}^{t}\right)^{t}, \mathbf{C}_{\mathbf{c o}}=\left(\mathbf{C}_{\mathbf{c o}_{1}}, \ldots \mathbf{C}_{\mathbf{c o}_{\mathbf{p}}}\right)$, and $\mathbf{C}_{\mathbf{o b}}=$ $\left(\mathbf{C}_{\mathbf{o b}_{\mathbf{1}}}, \ldots \mathbf{C}_{\mathbf{o b}_{\mathbf{p}}}\right)$, with, for $i=1, \ldots, p$,

$$
\mathbf{A}_{\mathbf{i}}=\left[\begin{array}{cc}
-2 \zeta_{i} \omega_{i} & -\omega_{i} \\
\omega_{i} & 0
\end{array}\right]
$$

$$
\begin{aligned}
\mathbf{B}_{\mathbf{i}} & =\left[\begin{array}{c}
\mathbf{b}_{\mathbf{i}} \\
\mathbf{0}
\end{array}\right] \\
\mathbf{C}_{\mathbf{c o}_{\mathbf{i}}} & =\left[\begin{array}{ll}
\mathbf{0} & \frac{\mathbf{c}_{\mathbf{c o}_{\mathbf{i}}}}{\omega_{i}}
\end{array}\right] \\
\mathbf{C}_{\mathbf{o b}_{\mathbf{i}}} & =\left[\begin{array}{ll}
\mathbf{0} & \frac{\mathbf{c}_{\mathrm{ob}_{\mathbf{i}}}}{\omega_{i}}
\end{array}\right]
\end{aligned}
$$

where $\mathbf{b}_{\mathbf{i}}, \mathbf{c}_{\mathbf{c o}_{\mathbf{i}}}$, and $\mathbf{c}_{\mathbf{o} \mathbf{b}_{\mathbf{i}}}$ are the $i^{\text {th }}$ components of $\boldsymbol{\Psi}^{t} \mathbf{E}_{\mathbf{g}}$, $\mathbf{F}_{\mathbf{g}} \Psi$ and $\mathbf{L}_{\mathbf{g}} \Psi$ respectively. Matrix $\mathbf{A}$ revolves on the structural parameters (eigen frequencies and damping ratio), whereas matrix $\mathbf{B}$ depends on the location of actuated DOF, matrix $\mathbf{C}_{\mathbf{c o}}$ on the location of desired displacement output, and matrix $\mathbf{C}_{\mathrm{ob}}$ on the location of integrated piezoelectric sensors. Fig. 3 shows the control-observation diagram principle to be applied on our system.

\section{B. Computation of the Observability and Balanced Grami-} ans

Observability gramian $\left(\mathbf{W}_{\mathbf{o b}}\right)$ between state $\mathbf{x}$ and measured output $\mathbf{q}_{\mathrm{g}}$ is found to be convenient to characterize the modes' observability by the mean of electric charge $\mathbf{q}_{\mathrm{g}}$. Its energetic and geometric interpretations are demonstrated in [6] and [15]. For stable $\mathbf{A}, \mathbf{W}_{\mathbf{o b}}$ is obtained from algebraic solution of following Lyapunov equation:

$$
\mathbf{A}^{t} \mathbf{W}_{\mathbf{o b}}+\mathbf{W}_{\mathbf{o b}} \mathbf{A}+\mathbf{C}_{\mathbf{o b}}^{t} \mathbf{C}_{\mathbf{o b}}=\mathbf{0}
$$

Assuming that the damping ratios are infinitely small and the natural frequencies well spaced, which is widely accepted for flexible structures, the block diagonal forms of $\left(\mathbf{A}, \mathbf{C}_{\mathbf{o b}}\right)$ couple can be exploited to give closed-form analytical solution for the expression of the modal observability gramian [16]. It is diagonal and equal to:

$$
\mathbf{W}_{\mathrm{ob}}=\operatorname{diag}\left(\mathbf{W}_{\mathrm{ob}_{11}}, \ldots, \mathbf{W}_{\mathrm{ob}_{\mathrm{pp}}}\right)
$$

with, for $i=1, \ldots, p$,

$$
\mathbf{W}_{\mathbf{o b}_{\mathbf{i i}}}=\frac{\gamma_{q_{i i}}}{4 \xi_{i} \omega_{i}^{3}} \mathbf{I}_{\mathbf{2}}=\alpha_{i} \mathbf{I}_{\mathbf{2}}
$$

where $\gamma_{q_{i i}}=\mathbf{c}_{\mathbf{o b}_{\mathbf{i}}}^{t} \mathbf{c}_{\mathbf{o b}}$, and $\mathbf{I}_{\mathbf{2}}$ is the $2 \times 2$ identity matrix. For a given mode $\left(\xi_{i}, \omega_{i}\right), \gamma_{q_{i i}}$ scalars represent the way the $i^{\text {th }}$ mode is seen through the piezoelectric sensor blocks.

On the other hand, the observer should be able to reconstruct the dominant modes of $\delta / \mathbf{u}$ transfer by measuring $\mathbf{q}_{\mathrm{g}}$ (see Fig. 4). These dominant modes are symbolized by high Hankel singular values (HSVs) defining the balanced gramian $\mathbf{W}_{\mathbf{e} \delta}$ of $\left(\mathbf{A}, \mathbf{B}, \mathbf{C}_{\mathbf{c o}}\right)$ system as follows:

$$
\mathbf{W}_{\mathbf{c} \delta}=\mathbf{W}_{\mathbf{o} \delta}=\mathbf{W}_{\mathbf{e} \delta}=\operatorname{diag}\left(\sigma_{i}\right)
$$

where $\sigma_{\mathbf{i}}$ are the HSVs of the $\left(\mathbf{A}, \mathbf{B}, \mathbf{C}_{\mathbf{c o}}\right)$ system.

Note that balanced gramian is a useful tool for quantifying the joint controllability and observability of the system. It is shown that when the damping ratios decrease to zero, the physical modal state coordinates are approximately balanced in this asymptotic situation, and the approximate $i^{\text {th }}$ Hankel singular value for flexible structure is given by [16]

$$
\sigma_{i}=\frac{\sqrt{\mathbf{c}_{\mathbf{c o}_{\mathbf{i}}^{t}} \mathbf{c}_{\mathbf{c o}_{\mathbf{i}}} \mathbf{b}_{\mathbf{i}} \mathbf{b}_{\mathbf{i}}^{t}}}{4 \xi_{i} \omega_{i}^{2}}
$$




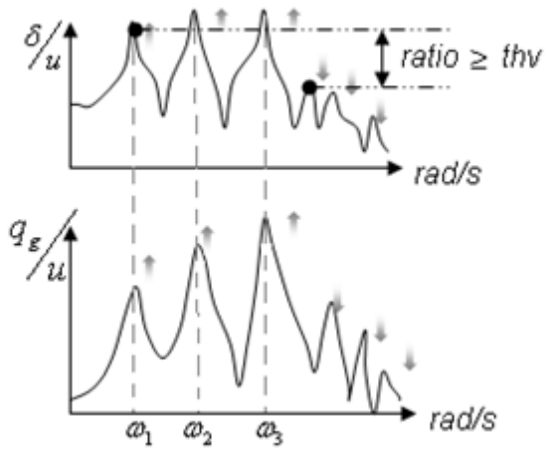

Fig. 4. Example of desired form of Control and Observation transfers: corresponding good control authority and good observability of the first $k=3$ modes

HSV describes the degree of the corresponding modal state's input-output energy flow through the system.

C. A Gramian-Based Criterion for the Optimization of the Actuator and Sensor Placement Within a Piezoelectric Micromechanism

Considering the statements made in Section III.B, this kind of active structures which are to be finely controlled are confronted with two main issues:

1) A reduced model of the structure must be developed, which includes the few dominant low frequency modes, without destabilizing the system by rejecting the residual modes (i.e. high roll-off after the dominant modes).

2) If the dominant modes are not all observed, the reconstruction of $\hat{\delta}$ will not be guarenteed in an optimal way by the observer.

As mentioned earlier, each piezostructure suggested in the optimization procedure is evaluated according to the specified criteria. Hence, we developed a new criterion to help overcome these two difficulties: the first problem is conquered by forcing the optimal structure to have $k$ first dominant modes of the $\delta / \mathbf{u}$ transfer in order to reduce the model without destabilizing it. Then, to surmount the second problem, we guarantee a high-level of observability of these dominant modes by means of electric charge $q_{g}$. It is thus presented by a procedure formulated as follows:

$$
\begin{gathered}
\text { If } \frac{\sigma_{i=1 \rightarrow k}^{\min }}{\sigma_{j=k+1 \rightarrow p}^{\max }} \geq t h v, \\
\text { then } \mathbf{J}^{k, n, t h v}=\frac{\sigma_{i=1 \rightarrow k}^{\min }}{\sigma_{j=k+1 \rightarrow p}^{\max }} \cdot \sum_{i=1}^{k} \alpha_{i}\left(\frac{\sigma_{i}}{\sigma_{j=1 \rightarrow k}^{\max }}\right)^{n}
\end{gathered}
$$

where $k$ is the number of the first dominant modes specified by the designer, $\sigma_{i=1 \rightarrow k}^{\min }$ and $\sigma_{j=k+1 \rightarrow p}^{\max }$ are respectively the level of the least dominant mode within the first $k$ and the level of the most dominant mode within the residual ones. $t h v$ is a threshold value specified by the designer. Hence, the condition (15) represents the domination of the $k$ first modes by at least $t h v$ times compared to the residual modes (see Fig. 4). It is inspired by a singular-value-decomposition based truncation method, called balanced model reduction introduced by [17]. Furthermore, in the numerical expression (16) of the criterion itself, $\alpha_{i}=\frac{\gamma_{q_{i i}}}{4 \xi_{i} \omega_{i}^{3}}$ corresponds to the coefficient of the $i^{\text {th }}$ observability modal gramian $\mathbf{W}_{\mathbf{o b}_{\mathbf{i i}}}$. $\left(\sigma_{i} / \sigma_{j}^{\text {Max }}\right)^{n}$ is a weighing ratio $\in[\mathbf{0}, \mathbf{1}]$. When the latter's value is close to 1 , the $i^{\text {th }}$ mode in question is a dominant mode within the first $k$ ones. Note that the exponant $n$ helps emphasizing the most dominant modes by accelerating the convergence towards $\sigma_{i}^{M a x}$. Thus, the corresponding $\alpha_{i}$ is privileged compared to other modes' observability. In other words, by maximizing this criterion, we privilege the modes where good observability of $\delta$ coincides with its dominant modes (Fig. 4). In this way, it appears that the procedure given by (15) and (16) is able to solve the problems mentioned above.

\section{MULTIOBJECTIVE OPTIMAL SYNTHESIS OF PIEZOELECTRIC MECHANISM WITH INTEGRATED ACTUATOR AND SENSOR}

\section{A. Optimization Problem Specifications}

We consider here the synthesis of a monolithic micromechanism with integrated actuator and sensor, made of a single piezoelectric material PIC151 from PI Piezo Ceramic Technology [18].

The structure topology is considered to have a maximal size of $15 \mathrm{~mm} \times 18 \mathrm{~mm}$, and a constant thickness of $200 \mu \mathrm{m}$. It is defined to be made of either passive, active or sensitive blocks inside a $2 \times 3$ mesh (see Fig. 5). For the optimal synthesis run, the number of active (resp. sensitive) blocks in the structure, chosen among blocks given in Fig. 1, is chosen to vary between 1 and 4 . When external voltages are applied to the blocks electrodes, the chosen output node of the structure has to move along the $x$-axis. For evaluation of static mechanical criteria, the potential difference between upper and lower face is taken equal to $200 \mathrm{~V}$. The size ratio of the blocks can vary as $b_{\max } / b_{\min } \in \llbracket 1 ; 2 \rrbracket$ and $a_{\max } / a_{\min } \in \llbracket 1 ; 2 \rrbracket$ (see Fig. 5 for details about $a$ and $b$ parameters definitions). The number of blocked nodes is comprised between 1 and 3 among the locations permitted which are reported in Fig. 5.

Finally, two numerical criteria to be maximized with FlexIn are:

- $J^{1}$ : free mechanical displacement $\delta_{\mathbf{x}}$ at the output node in $\mathrm{x}$-direction,

- $J^{2}$ : amplitude of the sensing electric charges induced on the piezoelectric blocks [10].

Note that $J^{1}$ and $J^{2}$ consider only the static behavior of the structure. They are calculated via the finite element approach used in FlexIn.

Simultaneously, a third dynamic criterion presented in section III.C is evaluated along with the first two:

- $J^{k, n, t h v}$ : modal observability of the mechanism output $\delta$ by the observed sensitive blocks charges $\mathbf{q}_{\mathrm{g}}$. In this example, we chose $k=2, n=2$ and $t h v=3$. 


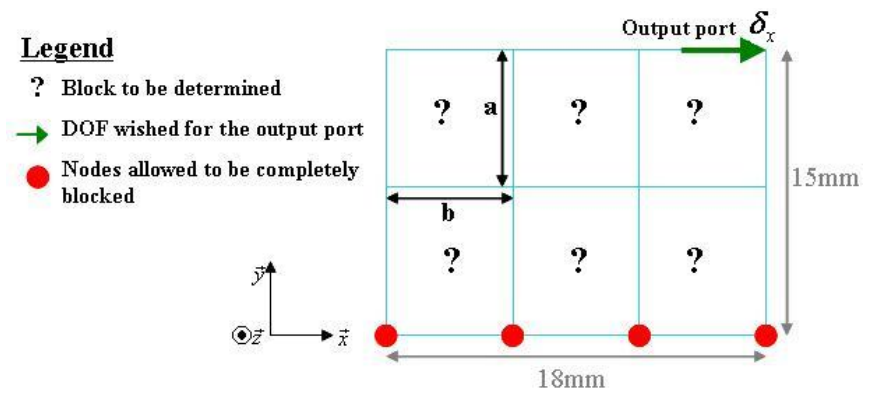

Fig. 5. Mesh of the PZT compliant micromechanism with imposed and permitted boundary conditions. $a$ and $b$ optimization parameters define the relative height and width of the blocks.
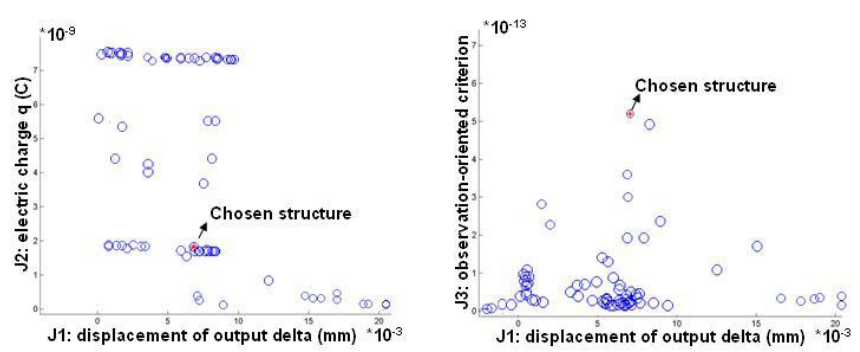

Fig. 6. Pareto fronts of compliant mechanisms synthesized using FlexIn.

\section{B. Results and Validation}

After defining the problems specifications, the optimization is set to run. When the genetic algorithm does not find any new pseudo-optimum during 200 subsequent generations, the best compromises are kept and can be found on Pareto fronts (Fig. 6). The designer can chose among these solutions, after studying their static and dynamic behaviours. In our case, the structure chosen appears to have the best dynamic desired behavior according to $J^{3}$, while static criteria remain very acceptable. It allows a tip displacement of $7 \mu \mathrm{m}$, and induces sensing charges around $1.9 \times 10^{-9} \mathrm{C}$. To illustrate the dynamic behavior, bode diagrams of $\delta / \mathbf{u}$ control transfer and $\mathbf{q}_{\mathrm{g}} / \mathbf{u}$ observation transfer given by FlexIn and commercial $3 D$ finite element Comsol software are shown in Fig. 7.b. The slight error between the resonant frequencies is mainly due to the fact that the building block 6 present in the structure is replaced by full triangles for machining conveniences, as shown in Fig. 7.a .

\section{Control of the Chosen Structure in Simulation}

Considering the modal state-space representation (5), we chose to reduce it to the first two dominant modes as mentioned earlier. Thus, from bode diagrams given by Comsol software in Fig. 7.b, open-loop $\delta / \mathbf{u}$ control transfer and $\mathbf{q}_{\mathrm{g}} / \mathbf{u}$ observation transfer are both identified, considering the first two dominant modes as follow:

$$
\begin{aligned}
& G_{c o}(s)=\frac{\delta(s)}{u(s)}=\frac{k_{c 1}}{\frac{s^{2}}{\omega_{1}^{2}}+\frac{2 \zeta_{c 1} s}{\omega_{1}}+1}+\frac{k_{c 2}}{\frac{s^{2}}{\omega_{2}^{2}}+\frac{2 \zeta_{c 2} s}{\omega_{2}}+1} \\
& G_{o b}(s)=\frac{q(s)}{u(s)}=\frac{k_{o 1}}{\frac{s^{2}}{\omega_{1}^{2}}+\frac{2 \zeta_{o 1} s}{\omega_{1}}+1}+\frac{k_{o 2}}{\frac{s^{2}}{\omega_{2}^{2}}+\frac{2 \zeta_{o 2} s}{\omega_{2}}+1}
\end{aligned}
$$
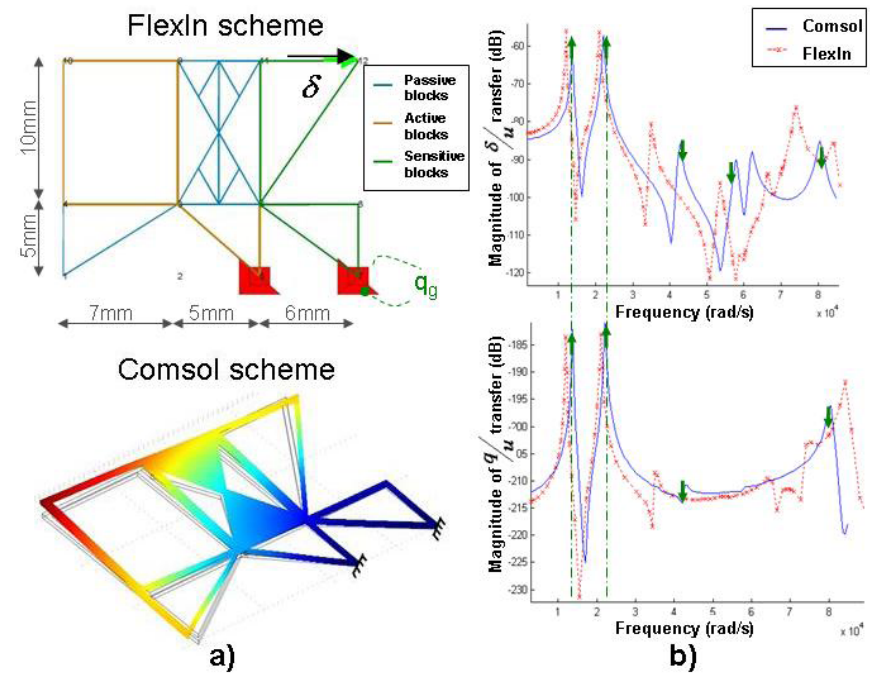

b)
Fig. 7. a) FlexIn and Comsol schemes of the chosen structure, b) Bode diagrams of control and observation transfers of the chosen structure.

Note that since flexible structures are slightly damped, we assume that all damping ratios are equal $\left(\zeta_{c i}=\zeta_{o i}=2 \%\right)$, which simplifies by far the computation of the modal statespace representation. Furthermore, the purpose of our optimization criterion $J^{3}$ is successfully achieved, which leads to the same natural frequencies in both transfer functions $\left(\omega_{1}=1.37 \times 10^{4} \mathrm{rad} / \mathrm{s}\right.$ and $\left.\omega_{2}=2.21 \times 10^{4} \mathrm{rad} / \mathrm{s}\right)$. Numerical values of identified static gains of both transfer functions are mentioned in Table I.

After specifying the state vector as in (4), we apply the same process followed in section III.A, where $p=2$ is the number of flexible modes. Finally, for values from (7) to (9), we obtain $\mathbf{b}_{\mathbf{i}}=\omega_{i}^{2}, \mathbf{c}_{\mathbf{c o}_{\mathbf{i}}}=k_{c i}$ and $\mathbf{c}_{\mathbf{o b}_{\mathbf{i}}}=k_{o i}$.

To make the model more realistic, we introduce white noises $\mathbf{v}(\mathbf{t})$ and $\mathbf{w}(\mathbf{t})$ applied respectively on the state $\mathbf{x}$ and the measured output $\mathbf{q}_{\mathrm{g}}$. In the following, two steps are performed independently, according to the separation principle: we first synthesize a Kalman filter to reconstruct the state via the measurement of $\mathbf{q}_{\mathrm{g}}$ and to minimize the noise effect on the control. Then, we implement a reconstructed modal state feedback control strategy.

1) Kalman filter estimator: it is known for its accuracy to optimally estimate the states in the presence of disturbances. In our case, we specified the noises' variances $\mathbf{V}(t)$ and $\mathbf{W}(t)$ to bring approximately $10 \%$ error on the state and measurement vectors respectively. The state reconstruction is given by :

$$
\begin{gathered}
\dot{\hat{\mathbf{x}}}(t)=\mathbf{A} \hat{\mathbf{x}}(t)+\mathbf{B u}(t)+\mathbf{K}_{\mathbf{o b}}\left(\mathbf{y}_{\mathbf{o b}}(t)-\mathbf{C}_{\mathbf{o b}} \hat{\mathbf{x}}(t)\right) \\
\mathbf{K}_{\mathbf{o b}}=\mathbf{\Sigma}(t) \mathbf{C}_{\mathbf{o b}}{ }^{T} \mathbf{W}(t)^{-1},
\end{gathered}
$$

where $\boldsymbol{\Sigma}(t)$ the reconstruction error variance, is the solution of Riccati equation:

$$
\mathbf{A} \boldsymbol{\Sigma}(t)+\boldsymbol{\Sigma}(t) \mathbf{A}^{T}+\mathbf{V}(t)-\boldsymbol{\Sigma}(t) \mathbf{C}_{\mathbf{o b}}{ }^{T} \mathbf{W}(t)^{-1} \mathbf{C}_{\mathbf{o b}} \boldsymbol{\Sigma}(t)=0
$$

2) Reconstructed state feedback control strategy: It is a very common control strategy based on pole placement 
TABLE I

IDENTIFIED PARAMETERS OF $G_{C O}$ AND $G_{o b}$ TRANSFER FUNCTIONS

\begin{tabular}{lc|cc}
\hline \hline \multicolumn{2}{c|}{$G_{c o}$} & \multicolumn{2}{|c}{$G_{o b}$} \\
\hline$k_{c 1}(\mathrm{~mm} / \mathrm{V})$ & $k_{c 2}(\mathrm{~mm} / \mathrm{V})$ & $k_{o 1}(\mathrm{C} / \mathrm{V})$ & $k_{o 2}(\mathrm{C} / \mathrm{V})$ \\
\hline $3.40 \times 10^{-5}$ & $2.76 \times 10^{-5}$ & $-1.59 \times 10^{-11}$ & $-9.30 \times 10^{-12}$ \\
\hline
\end{tabular}

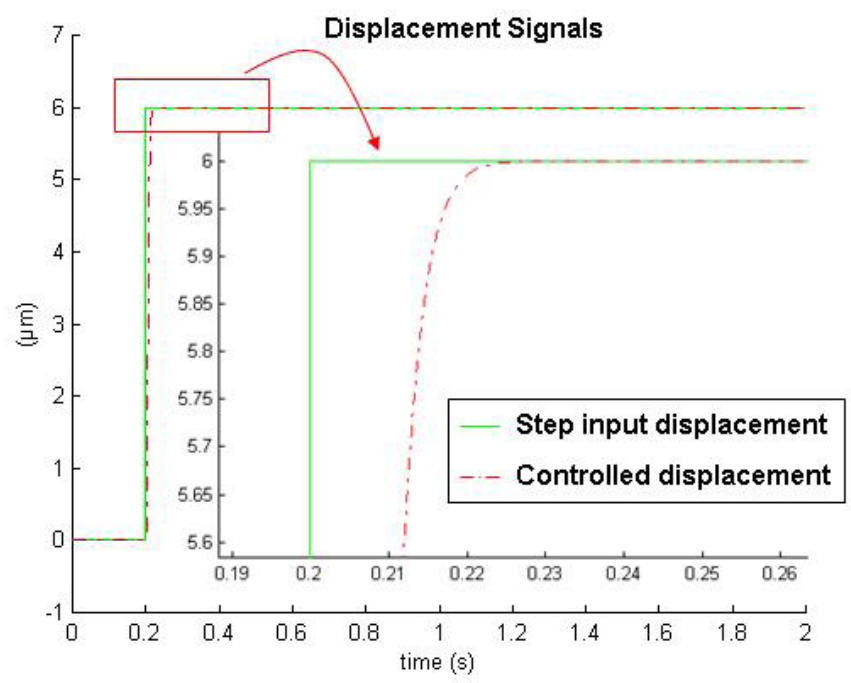

Fig. 8. Step response of the controlled structure.

by state feedback control. The idea is to synthesize a full state feedback $\mathbf{u}=-\mathbf{F} \hat{\mathbf{x}}$, where the gain $\mathbf{F}$ is selected to achieve desirable properties of the closed-loop system. In our context, by defining the theoretical time response $t_{r}=10 \mathrm{~ms}$ and specifying a zero overshoot, we determine the poles of the closed loop transfer function designated by $(\mathbf{A}-\mathbf{B F})$ matrix. In this way, they are two double pole pairs with zero imaginary parts $\left(p_{1}=p_{2}=-500\right.$ and $\left.p_{3}=p_{4}=-800\right)$.

3) Response analysis: Fig. 8 presents the time response to a $6 \mu \mathrm{m}$ step input on the structure controlled by the pole placement strategy. From this plot, we can conclude that this strategy is satisfying in simulation, in term of noise rejection and overshoot reduction. The time response at $5 \%$ from reference is $12.5 \mathrm{~ms}$. Furthermore, the system shows very appealing stability margins: gain margin $=104.7 \mathrm{~dB}$ and infinite phase margin.

\section{CONCLUSION}

A contribution to an existing optimal compliant micromechanisms design method has been presented in this paper. In this method, structures composed of an assembly of building blocks made of PZT, optimally integrate active and sensing blocks by means of a control-observation oriented gramian-based criterion. This is achieved by considering the open-loop dynamic control and observation transfers' performances. In fact, for optimally integrating both actuation and sensing functions in the structure, the criterion implies matching high control authority modes from the balanced gramian matrix, with high observation ability from the observation gramian matrix.
An optimization problem is specified to illustrate the mentioned methodology. The analysis of the resulting pseudooptimal solution bring to satisfying conclusion: it can operate at both static and dynamic regimes, and more importantly, the dynamic properties successfully sought by our optimization gramian-based criterion, simplify the control of such structures as shown in the last part.

A prototype of the compliant pseudo-optimal micromechanism resulting from the optimal design will be developed for experimental validations and control.

\section{REFERENCES}

[1] X. Lin, F.G. Yuan, Diagnostic lamb waves in an integrated piezoelectric sensor/actuator plate: analytical and experimental studies, Smart Materials and Structures 10, 2001, pp 907-913.

[2] D. Campolo, R. Sahai, R.S. Fearing, "Development of piezoelectric bending actuators with embedded piezoelectric sensors for micromechanical flapping mechanisms", IEEE Int. Conf. on Robotics and Automation, Taipei, Taiwan, 2003, pp 3339-3346.

[3] P.J Costa Branco, J.A. Dente, On the electromechanics of a piezoelectric transducer using a bimorph cantilever undergoing asymmetric sensing and actuation, Smart Materials and Structures, vol. 13, 2004, pp 631-642.

[4] I.A. Ivan, M. Rakotondrabe, P. Lutz, N. Chaillet, Quasistatic displacement self-sensing method for cantilevered piezoelectric actuators, Review of Scientific Instruments 80, 2009, pp 065102-1 - 065102-8.

[5] X. Ma, W. Dong, X. Ding, P. Zhang, C. Yan, Piezoelectric self-sensing actuator for beam vibration control based on blind source seperation, Asian Meeting on Ferroelectrics No. 5, vol. 358, Noda Japon, 2007, pp 29-34.

[6] S. Leleu, H. Abou-Kandil, Y. Bonnassieux, Piezoelectric Actuators and Sensors Location for Active Control of Flexible Structures, IEEE Trans. on Instrumentation and Measurement, vol.50, No.6, 2001, pp. 1577-1582.

[7] M. Collet, L. Jezequel, Active modal control with piezoelectric layers optimisation, Journal of Structural Control, vol.1, issue 1-2, 1994, pp 59-77.

[8] J.H. Han, I. Lee, Optimal placement of piezoelectric sensors and actuators for vibration control of composite plate using genetic algorithms, Smart Materials and Structures 8, 1999, pp 257-267.

[9] P. Bernardoni, P. Bidaud, C. Bidard, F. Gosselin, A new compliant mechanism design methodology based on flexible building blocks, Smart Material and Structures, vol. 5383, USA, 2004, pp 244-254.

[10] R. El Khoury Moussa, M. Grossard, C. Rotinat-Libersa, M. Boukallel, N. Chaillet, A. Hubert, Méthode des blocs sensitifs pour la synthèse optimale de mécanismes flexibles à mesure piézoélectrique intégrée, Mécanique \& Industries, vol. 10, No. 3-4, 2009, pp 317-322.

[11] M. R. Kermani, M. Moallem, R. V. Patel, "Optimizing the performance of piezoelectric actuators for active vibration control", in Proc. of IEEE International Conference on Robotics and Automation, Washington DC, 2002, pp 2375-2380.

[12] M. Collet, Shape optimization of piezoelectric sensors dealing with spill-over instability, IEEE Trans. on Control Systems Technology, vol. 9, No. 4, 2001, pp 654-662.

[13] M. Grossard , C. Rotinat-Libersa, N. Chaillet, M. Boukallel, Mechanical and control-oriented design of a monolithic microgripper using a new topological optimization method, IEEE/ASME Transactions on Mechatronics, vol. 14, No. 1, 2009, pp 32-45.

[14] A. Hac, and L. Liu, Sensor and actuator location in motion control of flexible structures, J. Sound Vib., vol. 167, No. 2, 1993, pp 239-261.

[15] E. A. Jonckheere, Principal components analysis of flexible systems, Open-loop case, IEEE Trans. Autom. Control, vol. AC-29, No. 12, 1984, pp 1095-1097.

[16] W. Gawronski and K. B. Lim, Balanced Control of Flexible Structures, London, U.K. Springer-Verlag; 1996.

[17] B. C. Moore, Principal component analysis in linear systems: controllability, observability, and model reduction, IEEE Trans. Automatic Control, vol.26, No. 1, 1981, pp 17-32.

[18] PI Piezo Ceramic Technology, Available: http://www.piceramic.com/.

[19] Lockheed Missiles and Space Company Inc., Vibration control of space structures: a high and low autorithy hardware implementation, AFWAL-TR-83-3074, July 1983. 